University of Wollongong

Research Online

Faculty of Engineering and Information

Faculty of Engineering and Information

Sciences - Papers: Part A

Sciences

$1-1-2015$

Maximising energy efficiency in antenna selection MIMO wireless systems subject to QoS constraints

Ngoc Phuc Le

University Of Wollongong, pnl750@uowmail.edu.au

Farzad Safaei

University of Wollongong, farzad@uow.edu.au

Le Chung Tran

University of Wollongong, Ictran@uow.edu.au

Follow this and additional works at: https://ro.uow.edu.au/eispapers

Part of the Engineering Commons, and the Science and Technology Studies Commons

Research Online is the open access institutional repository for the University of Wollongong. For further information contact the UOW Library: research-pubs@uow.edu.au 


\title{
Maximising energy efficiency in antenna selection MIMO wireless systems subject to QoS constraints
}

\author{
Abstract \\ Energy-efficiency (EE) in antenna selection multi-input-multi-output (MIMO) systems over Nakagami-m \\ fading channels is investigated. An EE metric is defined as the number of successfully received bits per \\ unit of energy consumption, which takes into account several important system parameters such as \\ channel coding and modulation. An optimisation problem that maximises the EE metric subject to an \\ error-performance constraint is formulated. On the basis of an analysis of this problem, the optimal value \\ of the average energy per transmitted data symbol is obtained, such that the EE in the system is \\ maximised. Simulation results are provided to validate the analysis.
}

\section{Keywords}

constraints, efficiency, qos, energy, maximising, subject, systems, wireless, mimo, selection, antenna

Disciplines

Engineering | Science and Technology Studies

\section{Publication Details}

N. P. Le, F. Safaei \& L. C. Tran, "Maximising energy efficiency in antenna selection MIMO wireless systems subject to QoS constraints," Electronics Letters, vol. 51, (16) pp. 1290-1292, 2015. 


\section{Maximising energy efficiency in antenna selection MIMO wireless systems subject to QoS constraints}

\author{
N. P. Le, F. Safaei and L. C. Tran
}

In this letter, we investigate energy efficiency (EE) in antenna selection multi-input multi-output (MIMO) systems over Nakagami- $m$ fading channels. We define an EE metric as the number of successfully received bits per unit energy consumption, which takes into account several important system parameters such as channel coding and modulation. An optimisation problem that maximises the EE metric subject to an errorperformance constraint is formulated. Based on an analysis of this problem, we obtain the optimal value of the average energy per transmitted data symbol such that the energy efficiency in the system is maximised. Simulation results are provided to validate the analysis.

Introduction: Antenna selection (AS) is a simple but powerful multiinput multi-output (MIMO) technique, as it could attain the benefits of full MIMO systems with only a small number of radio frequency (RF) chains [1]. Traditionally, the efficacy of AS schemes is investigated from an error-performance or capacity perspective. Due to an increasing concern about energy consumption in future wireless networks, it is important to study energy efficiency in AS systems. Recently, some research works have considered the energy efficiency in AS systems, e.g. [2], [3]. However, the energy-efficiency (EE) metric in [2] and [3] is defined as a ratio between capacity and power consumption, which does not take into account many important system parameters, such as channel codes or modulation. In [4], we studied energy efficiency in antenna selection MIMO automatic repeat request (ARQ) systems, in which the defined EE metric of energy consumption per bit involves the aforementioned system parameters. However, the formulated problem in [4] is only applicable to a MIMO ARQ system with an unlimited number of retransmissions, which is different from the focus of the present work.

In this letter, we perform an analysis of energy efficiency in antenna selection MIMO systems over Nakagami- $m$ fading channels. Unlike the related works in the literature, this paper defines an EE metric as the number of successfully received bits per unit energy consumption. Our objective is to maximise the EE metric subject to an error-performance requirement. Based on a convexity analysis of this optimisation problem, the optimal value of the average energy per transmitted symbol is obtained such that the energy efficiency in the system is maximised.

System model: We consider an antenna selection MIMO system with $n_{T}$ transmit antennas and $n_{R}$ receive antennas over a quasi-static Nakagami$m$ fading channel. Each data frame consisting of $L$ bits is first encoded by a rate- $r_{c}$ channel encoder, and then mapped into an $M$-ary quadrature amplitude modulation ( $M$-QAM) constellation. At any time instant, only one out of $n_{T}$ transmit antennas and only one out of $n_{R}$ receive antennas are selected for data transmission. The channel coefficient between the $i^{\text {th }}$ transmit antenna and the $j^{\text {th }}$ receive antenna is denoted as $h_{i, j}, i=1,2, \ldots$, $n_{T}, j=1,2, \ldots, n_{R}$. Also, the amplitude $\left|h_{i, j}\right|$ follows a Nakagami distribution with a fading parameter $m$ and $\varepsilon\left\{\left|h_{i, j}\right|^{2}\right\}=1$, where $\varepsilon\{$.$\} denotes an$ expectation operator.

We consider a maximum channel gain criterion for the antenna selection operation in this work. Accordingly, the transmit and receive antennas are selected by

$$
(\hat{i}, \hat{j})=\arg \max _{i=1,2 . ., n_{T} ; j=1,2 . ., n_{R}}\left|h_{i, j}\right|^{2} .
$$

Let $\gamma$ denote the instantaneous post-processing signal-to-noise ratio (SNR). The expression of the cumulative distribution function (cdf) of $\gamma$ can be expressed as [4]

$$
F_{\bar{\gamma}}(\gamma)=\left(1-e^{-m \gamma / \bar{\gamma}} \sum_{k=0}^{m-1}(m \gamma / \bar{\gamma})^{k} / k !\right)^{n_{T} n_{R}}, \gamma \geq 0,
$$

where $\bar{\gamma}$ is the average SNR that is defined as

$$
\bar{\gamma}=\frac{E_{s}}{G_{d} N_{0} r_{c} \log _{2} M},
$$

where $E_{s}$ denotes the average transmit energy per symbol, $N_{o}$ is the power spectral density of the additive white Gaussian noise at each receiver antenna, and $G_{d}$ is a factor that represents antenna gain, path-loss, and noise figure, etc. This factor can be expressed as $G_{d}=G_{0} d^{\chi} G_{M}$, where $G_{0}$ is the gain factor at the unit distance, $d$ is a transmission distance, $\chi$ is the path-loss exponent, and $G_{M}$ stands for other parameters such as noise figure and the link margin compensating the variations of hardware process [5].

Optimal transmit energy for maximal energy efficiency: We consider the number of successfully received data bits per unit energy consumption as a metric to measure the energy efficiency, i.e.

$$
E E=N_{b} /\left(N \times E_{\text {total }}\right), \quad(\text { bits } / \text { Joule })
$$

where $N_{b}$ is the number of successfully received data bits when we transmit $N$ data frames and $E_{\text {total }}$ is the total energy consumption at both transmitter and receiver corresponding to one data frame.

Assuming that a data frame is accepted and sent for upper-layer processing only if there is no bit-error within that frame, we can express

$$
N_{b}=L \times N \times(1-F E R), \quad(\text { bits })
$$

where FER is the average frame-error rate (FER). We note that a derivation of the exact expression for FER over quasi-static fading channels is difficult. However, an approximated FER can be obtained by using a threshold-based approximation approach [6], i.e. (cf. Eq. (2))

$$
F E R(\bar{\gamma}) \approx F_{\bar{\gamma}}\left(\gamma_{t h}\right)=\left(1-e^{-m \gamma_{t h} / \bar{\gamma}} \sum_{k=0}^{m-1}\left(m \gamma_{t h} / \bar{\gamma}\right)^{k} / k !\right)^{n_{T} n_{R}},
$$

where $\gamma_{t h}$ is the SNR threshold that can be estimated by using a minimum sum-error criterion or fitting (6) to the simulated FER curve.

The total energy consumption per frame $E_{\text {total }}$ can be expressed as

$$
E_{\text {total }}=L \times\left(E_{t} / r_{c} \log _{2} M\right),
$$

where $E_{t}$ is the total energy consumption per symbol that consists of the actual transmitted energy $E_{s}$ and the energy consumed by hardware $E_{c}$, i.e. $E_{t}=E_{s}+E_{c}$. Also, the energy $E_{c}$ is given as $E_{c}=(\xi / \eta-1) E_{s}+P_{c} / R_{s}$ [5], where $\eta$ is the drain efficiency of the power-amplifier (PA), $\xi \approx 3(\sqrt{M}-1 / \sqrt{M}+1), M \geq 4$, is the peak-to-average power ratio of $M$ QAM signals, $P_{c}$ is a power consumption of baseband processing units and RF chains at both transmitter and receiver (excluding the PA), and $R_{s}$ is the symbol rate. Thus, $E_{\text {total }}$ can be rewritten as (cf. Eq. (3))

$$
E_{\text {total }}=L \times \frac{1}{r_{c} \log _{2} M}\left(\frac{\xi}{\eta} E_{s}+\frac{P_{c}}{R_{s}}\right):=L \times(\alpha \bar{\gamma}+\beta),
$$

where $\alpha=G_{d} N_{0}(\xi / \eta), \beta=P_{c} / R_{b}$, and $R_{b}=R_{s} r_{c} \log _{2} M$ is the data bit rate. Substituting (5), (6), and (8) into (4) results in

$$
E E(\bar{\gamma})=\frac{1-\left(1-e^{-m \gamma_{t h} / \bar{\gamma}} \sum_{k=0}^{m-1}\left(m \gamma_{t h} / \bar{\gamma}\right)^{k} / k !\right)^{n_{T} n_{R}}}{\alpha \bar{\gamma}+\beta} .
$$

The energy efficiency maximisation problem can be now formulated as

$$
\begin{aligned}
& \text { maximise } \operatorname{EE}(\bar{\gamma}) \\
& \text { subject to } F E R(\bar{\gamma}) \leq F E R_{0},
\end{aligned}
$$

where $F E R_{0}$ is the required frame-error rate. By assuming that bit-errors are uncorrelated, we have $F E R_{0}=1-\left(1-B E R_{0}\right)^{L}$, where $B E R_{0}$ is the required bit-error rate.

Proposition 1: The energy-efficiency metric EE $(\bar{\gamma})$ defined in Eq. (9) is a quasi-concave function with respect to $\bar{\gamma}$. Also, the optimal value $\bar{\gamma}_{m}$ that maximises $E E(\bar{\gamma})$ is the root of the equation $\partial E E(\bar{\gamma}) / \partial \bar{\gamma}=0$.

Proof: The proof of the first part is similar to the proof of Theorem 1 in [4]. As a sketch of the proof, if $f(x)$ is a sigmoid function (or S-shaped), then $f(x) / x$ is a quasi-concave function [7]. Thus, we need to 
show that the numerator of $E E(\bar{\gamma})$ satisfies all the properties of a Sshaped function derived in [7]. For the second part, as $E E(\bar{\gamma})$ is quasiconcave, it clear that $E E(\bar{\gamma})$ has a unique maximum value at $\bar{\gamma}_{m}$.

Note that due to the complexity of the function $\operatorname{EE}(\bar{\gamma})$, it is hard to derive the closed-form expression for $\bar{\gamma}_{m}$. However, it is easy to numerically evaluate $\bar{\gamma}_{m}$, e.g. using Newton's method.

Proposition 2: There exists a positive value $\bar{\gamma}_{0}$ such that $\operatorname{FER}(\bar{\gamma}) \leq F E R_{0}$ when $\bar{\gamma} \geq \bar{\gamma}_{0}$. The value $\bar{\gamma}_{0}$ is the root of the equation $g(\bar{\gamma})=1-\left(F E R_{0}\right)^{1 / n_{T} n_{R}}$, where $g(\bar{\gamma})=e^{-m \gamma_{t h} / \bar{\gamma}} \sum_{k=0}^{m-1}\left(m \gamma_{t h} / \bar{\gamma}\right)^{k} / k !$

Proof: We can rewrite Eq. (6) as $F E R(\bar{\gamma})=(1-g(\bar{\gamma}))^{n_{T} n_{R}}$. Thus, the constraint of $F E R(\bar{\gamma}) \leq F E R_{0}$ is equivalent to $g(\bar{\gamma}) \geq 1-\left(F E R_{0}\right)^{1 / n_{T} n_{R}}$. For notational convenience, let us denote $\Gamma=1-\left(F E R_{0}\right)^{1 / n_{T} n_{R}}$. As $0<\Gamma<1$, we need to show that $g(\bar{\gamma})$ is an increasing function within an interval $(0,1)$. This is indeed the case given that $\partial g(\bar{\gamma}) / \partial \bar{\gamma}=\left(m \gamma_{t h}\right)^{m} e^{-m \gamma_{t h} / \bar{\gamma}} / \bar{\gamma}^{m+1}(m-1) !>0, \forall \bar{\gamma}>0, \lim _{\bar{\gamma} \rightarrow 0} g(\bar{\gamma})=0 \quad$ and $\lim _{\bar{\gamma} \rightarrow+\infty} g(\bar{\gamma})=1$. This completes the proof.

We note that when $m=1$ (i.e. a Rayleigh fading channel), the explicit expression of $\bar{\gamma}_{0}$ is given as $\bar{\gamma}_{0}=\gamma_{t h} / \log _{e}(1 / \Gamma)$. Also, when $m=2$, we have $\bar{\gamma}_{0}=-2 \gamma_{t h} /\left(W_{-1}(-\Gamma / e)+1\right)$, where $W_{\mathrm{k}}($.$) is the k^{\text {th }}$ branch of the Lambert $W$ function, and $e$ is the Euler's number, i.e. $e \approx 2.71828$. For other values of $m$, the value $\bar{\gamma}_{0}$ can be evaluated numerically.

The solution $\bar{\gamma}^{\text {opt }}$ of the problem (10) can now be obtained as follow.

Theorem 1: If the optimal value $\bar{\gamma}_{m}$ satisfies $F E R\left(\bar{\gamma}_{m}\right) \leq F E R_{0}$, it will be the solution to the problem (10), i.e. $\bar{\gamma}^{\text {opt }}=\bar{\gamma}_{m}$. Otherwise, $\bar{\gamma}^{\text {opt }}=\bar{\gamma}_{0}$.

Proof: The result is obtained directly from Propositions 1 and 2.

Once the optimal value $\bar{\gamma}^{\text {opt }}$ is calculated, we can get the optimal average energy per transmitted symbol by using Eq. (3), i.e.

$$
E_{s}^{o p t}=\bar{\gamma}^{o p t} G_{d} N_{0} r_{c} \log _{2} M
$$

Simulation results: We use the following parameters in our simulations, most of which follow those in [5]. In particular, $L=2000$ bits, $B E R_{0}=$ $10^{-4}, \eta=0.35, P_{c}=310 \mathrm{~mW}, N_{o} / 2=-174 \mathrm{dBm} / \mathrm{Hz}, G_{0}=30 \mathrm{~dB}, G_{M}=40$ $\mathrm{dB}, m=2, \chi=3.5, R_{b}=20 \mathrm{kbps}$, 4-QAM modulation, and a convolutional code with a rate $r_{c}=1 / 2$ and generator polynomial [5,7]8.

Fig. 1 plots the energy efficiency $E E(\bar{\gamma})$ versus the average SNR $\bar{\gamma}$. First, it can be seen that the analytical curves based on the FER approximation in Eq. (6) agree well with the simulation curves. Second, the AS system outperforms the single-input single-output (SISO) system from an EE perspective. Moreover, the energy efficiency is improved when the number of antennas is increased. This is because a larger diversity gain leads to a lower FER, which in turn improves energy efficiency. Note that in the high SNR regime, the FER values becomes very small. Also, the transmitted energy $E_{s}$ dominates the circuit energy consumption $E_{c}$. Hence, the EE value is asymptotic to $f(\bar{\gamma})=1 / \alpha \bar{\gamma}$ (cf. Eq. (9)). Third, the average SNR values $\bar{\gamma}_{0}, \bar{\gamma}_{m}$, and $\bar{\gamma}^{\text {opt }}$ are lower when the number of antennas increases. Consequently, by employing more antennas, the optimal transmitted energy $E_{s}^{o p t}$ is reduced. In Fig. 2, we show the optimal energy efficiency $E E\left(\bar{\gamma}^{\text {opt }}\right)$ versus the transmission distance $d$. It can be seen that $E E\left(\bar{\gamma}^{\text {opt }}\right)$ decreases when the distance $d$ increases. This makes sense as a larger transmitted energy is required to compensate for the increasing path-loss so that the FER constraint is satisfied. However, we note that the AS system can achieve higher energy-efficiency than the SISO system in all scenarios.

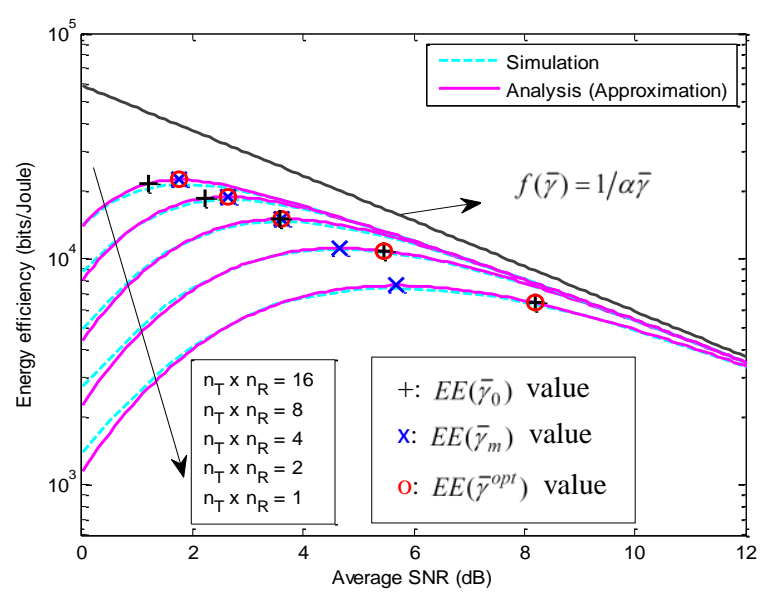

Fig. 1. Energy efficiency EE $(\bar{\gamma})$ versus the average SNR $\bar{\gamma}(d=100 m)$.

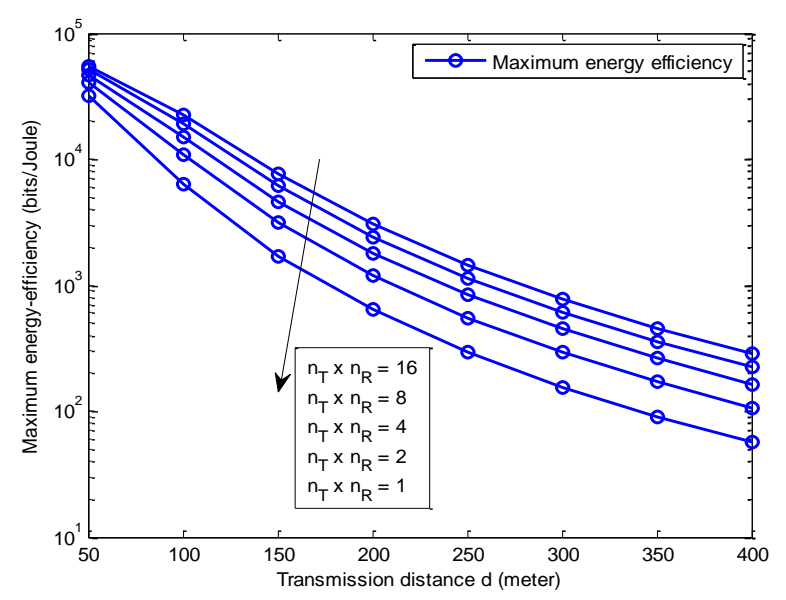

Fig. 2. Maximum energy efficiency versus the transmission distance.

Conclusions: This paper studies energy efficiency in antenna selection MIMO systems over Nakagami- $m$ fading channels. We consider the EE metric that takes into account several important system parameters such as channel coding and modulation, which is important from a practical viewpoint. We analyse the optimal value of the average energy per transmitted symbol such that the energy efficiency in the AS system is maximised. Our results show that the AS system offers a significant improvement in terms of energy efficiency, compared to the SISO system.

N. P. Le, F. Safaei and L. C. Tran (SECTE, University of Wollongong, NSW 2522, Australia)

E-mail:pn1750@uow.edu.au

\section{References}

1 Molisch, A.F., Win, M.Z.: 'MIMO systems with antenna selection', IEEE Microwave Magazine, 2004, 5, (1), pp. 46-56

2 Jiang, C., Cimini, L.J.: 'Antenna selection for energy-efficient MIMO transmission', IEEE Wireless Comm. Letters, 2012, 1, (6), pp.577-580

3 Zhou, X., Bai, B., Chen, W.: 'Iterative antenna selection for multistream MIMO under a holistic power model', IEEE Wireless Comm. Letters, 2014, 3, (1), pp. 82-85

4 Le, N.P., Tran, L.C., Safaei, F., Varma, V.S.: 'Energy-efficiency analysis of antenna selection MIMO ARQ systems over Nakagami- $m$ fading channels', IET Communications (accepted for publication, March, 2015)

5 Cui, S., Goldsmith A.J., Bahai, A.: 'Energy-constrained modulation optimization', IEEE Trans. Wireless Commu., 2005, 4, pp. 2349-2360

6 Chatzigeorgiou, I., Wassell, I.J., Carrasco, R.: 'On the frame error rate of transmission schemes on quasi-static fading channel'. Proc. 42nd Annual Conf. Infor. Sciences Systems., 2008, pp. 577-581

7 Rodriguez, V.: 'An analytical foundation for resource management in wireless communication', 2003 IEEE Globecom, pp. 898-902 